Research Article

\title{
Serum Autoantibodies against STIP1 as a Potential Biomarker in the Diagnosis of Esophageal Squamous Cell Carcinoma
}

\author{
Yi-Wei Xu, ${ }^{1,2,3}$ Can-Tong Liu, ${ }^{4}$ Xin-Yi Huang, ${ }^{4}$ Li-Sheng Huang, ${ }^{5}$ Yu-Hao Luo, ${ }^{1}$ \\ Chao-Qun Hong, ${ }^{6}$ Hai-Peng Guo, ${ }^{7}$ Li-Yan Xu, ${ }^{8}$ Yu-Hui Peng, ${ }^{1,3}$ and En-Min Li ${ }^{2,3}$ \\ ${ }^{1}$ Department of Clinical Laboratory Medicine, Cancer Hospital, Shantou University Medical College, Shantou 515041, China \\ ${ }^{2}$ Department of Biochemistry and Molecular Biology, Shantou University Medical College, Shantou 515041, China \\ ${ }^{3}$ The Key Laboratory of Molecular Biology for High Cancer Incidence Coastal Chaoshan Area of Guangdong Higher Education \\ Institutes, Shantou University Medical College, Shantou 515041, China \\ ${ }^{4}$ Shantou University Medical College, Shantou 515041, China \\ ${ }^{5}$ Department of Radiation Oncology, Cancer Hospital, Shantou University Medical College, Shantou 515041, China \\ ${ }^{6}$ Department of Oncological Research Laboratory, Cancer Hospital, Shantou University Medical College, Shantou 515041, China \\ ${ }^{7}$ Department of Surgical Oncology, Cancer Hospital, Shantou University Medical College, Shantou 515041, China \\ ${ }^{8}$ Institute of Oncologic Pathology, Shantou University Medical College, Shantou 515041, China
}

Correspondence should be addressed to Yu-Hui Peng; pengyuhui666@163.com and En-Min Li; nmli@stu.edu.cn

Received 9 May 2017; Revised 10 July 2017; Accepted 16 July 2017; Published 9 August 2017

Academic Editor: Stamatios E. Theocharis

Copyright (c) 2017 Yi-Wei Xu et al. This is an open access article distributed under the Creative Commons Attribution License, which permits unrestricted use, distribution, and reproduction in any medium, provided the original work is properly cited.

\begin{abstract}
Esophageal squamous cell carcinoma (ESCC) remains one of the leading causes of cancer-related mortality around the world. The identification of novel serum biomarkers is required for early detection of ESCC. This study was designed to elucidate whether autoantibodies against STIP1 could be a diagnostic biomarker in ESCC. An enzyme-linked immunosorbent assay was performed to detect serum levels of STIP1 autoantibodies in a training cohort (148 ESCC patients and 111 controls) and a validation cohort (60 ESCC patients and 40 controls). Mann-Whitney's $U$ test showed that ESCC patients in two cohorts have higher levels of autoantibodies against STIP1 when compared to controls $(P<0.001)$. According to receiver operating characteristic analysis, the sensitivity, specificity, and area under the curve (AUC) of autoantibodies against STIP1 in ESCC were 41.9\%, 90.1\%, and 0.682 in the training cohort and 40.0\%, 92.5\%, and 0.710 in the validation cohort, respectively. Moreover, detection of autoantibodies against STIP1 could discriminate early-stage ESCC patients from controls, with sensitivity, specificity, and AUC of $35.7 \%, 90.1 \%$, and 0.684 in the training cohort and $38.5 \%, 92.5 \%$, and 0.756 in the validation cohort, respectively. Our findings indicated that autoantibodies against STIP1 might be a useful biomarker for early-stage ESCC detection.
\end{abstract}

\section{Introduction}

Esophageal cancer (EC) is the eighth most prevalent malignant disease and the sixth leading cancer-related deaths around the world [1]. In China, 477,900 EC patients were diagnosed and 375,000 patients died in 2015. Among them, the number of male patients was as twice as that of female patients [2]. Esophageal squamous cell carcinoma (ESCC) and esophageal adenocarcinoma are two predominant subtypes of EC. In China, $90 \%$ of cases are ESCC, compared to only $26 \%$ in the United States [3]. Despite many advances in the treatments of patients with EC, the 5-year survival rate remains poor (e.g., $17.4 \%$ in the United States) [4]. The survival rate of EC could reach up to $85 \%$ when diagnosed at an early stage but is no more than $10 \%$ if diagnosed at an 
advanced stage [5]. Thus, early diagnosis offers a great opportunity to receive effective therapy and reduce ESCC mortality, and the discovery of noninvasive screening methods is urgently needed.

Serological tests are found to be one of the promising methods for improvement of the early detection of cancer. However, ESCC lacks effective and reliable serological biomarker for early detection and disease surveillance. Actually, carcinoembryonic antigen (CEA), squamous cell carcinoma antigen (SCCA), and CYFRA21-1 were the most widely used serum biomarkers for ESCC, but the performance of these biomarkers to detect early-stage ESCC is deficient [6-8]. Novel biomarkers with high diagnostic accuracy are greatly needed to improve detection of ESCC. In the last decade, numerous studies have indicated that autoantibodies against tumor-associated antigens (TAAs), as reporters from the immune system, exist in cancer patients, and autoantibodies against TAAs are thought to be ideal targets as noninvasive serological tests for early detection of cancer [9-11]. Therefore, autoantibodies could be a valuable source of serum biomarkers used for identifying early ESCC.

Stress-induced phosphoprotein 1 (STIP1), also known as HOP, P60, STI1, and so forth, is a 66.2-kilodalton chaperone protein which plays important roles in stress and nonstress conditions. Its 2.0-kilobase-encoded mRNA was first isolated from the yeast Saccharomyces cerevisiae [12]. STIP1 is one of the cochaperones that are most extensively studied and contains three tetratricopeptide repeat (TPR) domains, which can simultaneously bind Hsp70 and Hsp90 [13, 14]. STIP1 was identified to be overexpressed in several kinds of cancers, such as colorectal carcinoma (CRC) [15], pancreatic cancer [16], cholangiocellular carcinoma (CCC) [17], ovarian cancer [18], and so on. Moreover, increased expression of STIP1 may indicate poor survival outcome in cancer patients [18, 19]. STIP1 was also identified as a TAA recognized by the humoral immune system by means of serological analysis of recombinant cDNA expression library approach [20]. A recent study showed that autoantibodies against STIP1 were significantly elevated in the serum levels of patients with ovarian cancer, compared with the normal controls [21]. However, no study has been conducted on STIP1 autoantibodies in esophageal cancer. The present study was then undertaken to investigate whether autoantibodies against STIP1 could be altered and used as a candidate diagnostic biomarker in ESCC.

\section{Methods}

2.1. Study Population. The serum samples of 148 patients with ESCC and 111 normal controls as a training cohort were collected from the Cancer Hospital of Shantou University Medical College, from March 2013 to June 2014. The sera of 40 normal controls and 60 ESCC patients obtained from the same hospital between July 2014 and February 2015 were used as a validation cohort. The sex and age were well matched in the patient group and control group in both cohorts (Table 1). ESCC patients were all newly diagnosed, and serum samples were obtained prior to any anticancer treatment. The normal controls must have no evidence of any malignancies based on physical examination. All patients and normal controls gave written informed consent to attend this study, which was approved by the institutional review board of the Cancer Hospital of Shantou University Medical College and conformed to the requirements of the Declaration of Helsinki.

ESCC was diagnosed and defined in our previous study $[22,23]$. We defined tumor stage in accordance with the Seventh Edition of the American Joint Committee on Cancer (AJCC) Cancer Staging Manual [24]. In the present work, ESCC with AJCC stage $0+$ I + IIA was treated as early-stage ESCC as reported [22].

2.2. Recombinant STIP1 Protein. The expression, purification, and analysis of the recombinant STIP1 proteins were conducted as previously described [22, 23]. Briefly, the coding sequence region of STIP1 (NM_006819) was subcloned into the pDEST17 vector system (Invitrogen). The recombinant plasmid was transformed into the expression host E. coli Rosetta (DE3; Novagen). Transformed colonies were inoculated and cultured overnight. Then, the cell culture was transferred to fresh LB medium. To induce expression of recombinant protein, IPTG (Merck) was added. Next, the cells were harvested and resuspended. Cell debris was cleared by centrifugation, and the supernatants were incubated by using a Ni2p-NTA-Sepharose column (Novagen). The proteins of interest were eluted and dialyzed. BCA protein assay (Thermo) was performed to determine protein concentrations with the use of bovine serum albumin as a standard. The purity of the recombinant protein was assessed by Coomassie Blue staining (Imperial Protein Stain; Thermo) following SDS-PAGE.

2.3. Enzyme-Linked Immunosorbent Assay (ELISA). ELISA was used to detect serum levels of autoantibodies against STIP1 by two investigators (Can-Tong Liu and Xin-Yi Huang) who were blinded to clinical information about ESCC patients and normal controls. ELISA protocol was conducted according to our prior work [22, 25]. In brief, recombinant STIP1 protein diluted to a concentration of $0.2 \mu \mathrm{g} / \mathrm{mL}$ was dispensed in $100 \mu \mathrm{L}$ per well volumes into 96-well microtiter plates and incubated overnight at $4^{\circ} \mathrm{C}$. The protein-coated wells were blocked with PBST containing $0.05 \%$ Tween-20 and $1 \%$ BSA at $37^{\circ} \mathrm{C}$ for $1 \mathrm{~h}$. After washing, serum samples and self-made quality control samples (a pooled serum sample randomly collected from 100 ESCC patients), all at the dilution of $1: 110$, were incubated at $37^{\circ} \mathrm{C}$ for another one hour, as well as appropriate polyclonal rabbit anti-STIP1 antibodies (Immunosoft, Zhoushan, China) specific for captured proteins. $100 \mu \mathrm{L}$ of secondary antibodies (i.e., goat anti-human/anti-mouse IgG-HRP) diluted at $1: 10,000$ was added into each well, followed by color development (ready-prepared 3,3',5,5'-tetramethylbenzidine and hydrogen peroxide). The measurement of optical density (OD) value of each well was completed on a microplate 
TABle 1: Participant details and clinicopathological features.

\begin{tabular}{|c|c|c|c|c|}
\hline \multirow{2}{*}{ Group } & \multicolumn{2}{|c|}{ Training cohort } & \multicolumn{2}{|c|}{ Validation cohort } \\
\hline & $\operatorname{ESCC}(n=148)$ & Normal $(n=111)$ & $\operatorname{ESCC}(n=60)$ & $\operatorname{Normal}(n=40)$ \\
\hline \multicolumn{5}{|l|}{ Age, years } \\
\hline Mean \pm SD & $58 \pm 9$ & $57 \pm 8$ & $59 \pm 6$ & $56 \pm 7$ \\
\hline Range & $41-88$ & $38-77$ & $43-75$ & $40-71$ \\
\hline \multicolumn{5}{|l|}{ Gender } \\
\hline Male & 114 & 69 & 38 & 26 \\
\hline Female & 34 & 25 & 22 & 14 \\
\hline \multicolumn{5}{|l|}{ Smoke } \\
\hline Yes & 106 & 73 & 36 & 29 \\
\hline No & 42 & 38 & 24 & 11 \\
\hline \multicolumn{5}{|l|}{ TNM stage } \\
\hline 0 & 3 & & 1 & \\
\hline $\mathrm{I}(\mathrm{IA}+\mathrm{IB})$ & $17(7+10)$ & & $8(3+5)$ & \\
\hline II (IIA + IIB) & $48(22+26)$ & & $20(4+16)$ & \\
\hline III (IIIA + IIIB + IIIC) & $76(32+14+30)$ & & $30(15+2+13)$ & \\
\hline IV & 4 & & 1 & \\
\hline \multicolumn{5}{|l|}{ Histological grade } \\
\hline High (grade 1) & 50 & & 17 & \\
\hline Middle (grade 2) & 84 & & 36 & \\
\hline Low (grade 3) & 14 & & 7 & \\
\hline \multicolumn{5}{|l|}{ Depth of tumor invasion } \\
\hline Tis & 3 & & 1 & \\
\hline $\mathrm{T} 1$ & 11 & & 10 & \\
\hline $\mathrm{T} 2$ & 19 & & 11 & \\
\hline T3 & 77 & & 20 & \\
\hline $\mathrm{T} 4$ & 38 & & 18 & \\
\hline \multicolumn{5}{|l|}{ Regional lymph nodes } \\
\hline N0 & 77 & & 31 & \\
\hline N1 & 38 & & 19 & \\
\hline $\mathrm{N} 2$ & 21 & & 9 & \\
\hline N3 & 12 & & 1 & \\
\hline \multicolumn{5}{|l|}{ Size of tumor } \\
\hline$<5 \mathrm{~cm}$ & 71 & & 24 & \\
\hline$\geq 5 \mathrm{~cm}$ & 77 & & 36 & \\
\hline \multicolumn{5}{|l|}{ Site of tumor } \\
\hline Upper thorax & 15 & & 10 & \\
\hline Middle thorax & 101 & & 46 & \\
\hline Lower thorax & 32 & & 4 & \\
\hline
\end{tabular}

reader (Multiskan MK3, Thermo Fisher Scientific, Boston) within $5 \mathrm{~min}$ at $450 \mathrm{~nm}$ with $630 \mathrm{~nm}$ reference.

All serum samples were tested in duplicate. Quality control for monitoring of intra-assay deviation or interassay deviation of the ELISA assay was conducted as described in our previous studies $[22,25]$. Briefly, the intra-assay and interassay coefficient of variations (CVs) for the ELISA method of the detection of autoantibodies against STIP1 were $7.9 \%$ and $9.4 \%$, respectively. Quality control samples were conducted to ensure quality control monitoring of the assay runs with the use of Levey-Jennings plots. To minimize an intra-assay deviation, the ratio of the difference between the duplicated sample OD values to their sum was used to evaluate assay precision. If the ratio was $>10 \%$, the measurement of this sample was deemed to be invalid and the sample was repeated.

2.4. Statistical Analysis. We performed all analyses by using Microsoft Excel, GraphPad Prism 5 software, and SPSS (version 17.0). The results of sample positive proportion and diagnostic parameters were shown with $95 \%$ exact confidence interval ( $95 \% \mathrm{CI})$ estimation. The differences of 


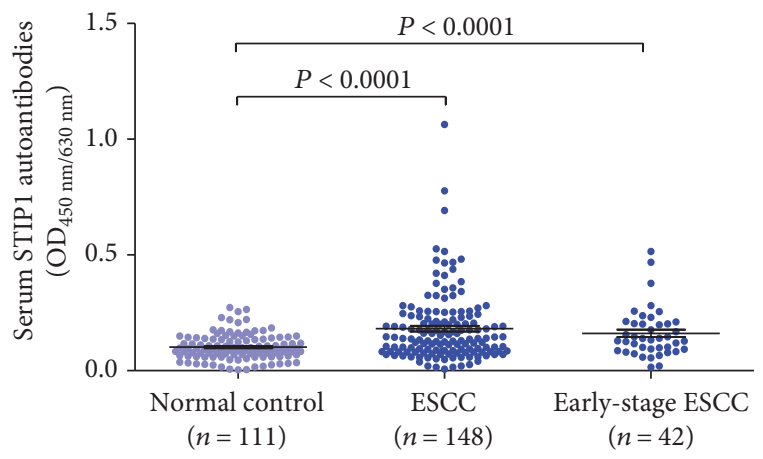

(a)

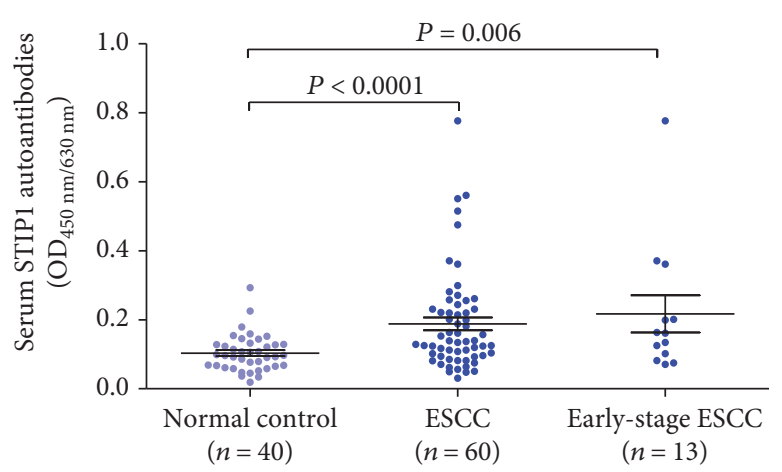

(c)

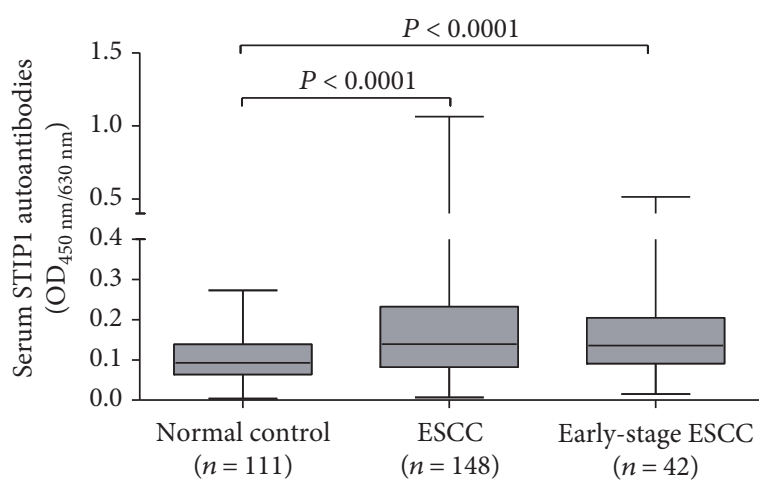

(b)

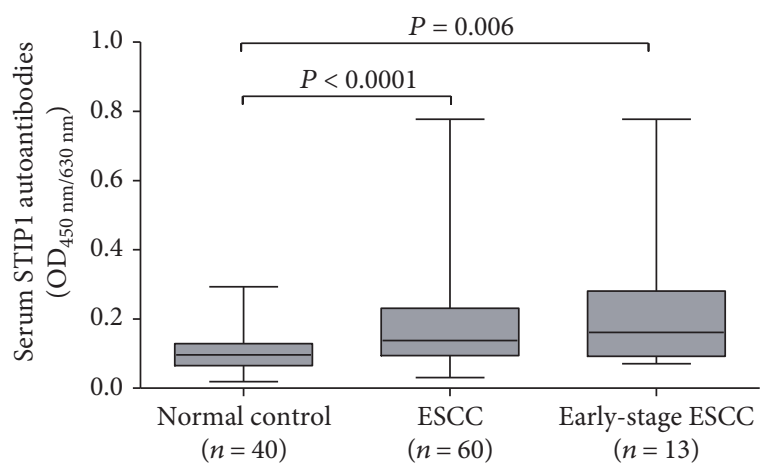

(d)

FIGURE 1: Levels of autoantibodies against STIP1 in ESCC. (a) Scatter plots of OD values of autoantibodies against STIP1 from sera of normal controls, ESCC patients, and early-stage ESCC patients in the training cohort. Black horizontal lines are means and error bars are SEs. (b) Median levels and interquartile ranges of serum autoantibodies against STIP1 in normal controls, ESCC patients, and early-stage ESCC patients in the training cohort are, respectively, illustrated by box plot, and the whiskers show minimum and maximum value. (c) Scatter plots of OD values of autoantibodies against STIP1 from sera of normal controls, ESCC patients, and early-stage ESCC patients in the validation cohort. Black horizontal lines are means and error bars are SEs. (d) Median levels and interquartile ranges of serum autoantibodies against STIP1 in normal controls, ESCC patients, and early-stage ESCC patients in the validation cohort are, respectively, illustrated by box plot, and the whiskers show minimum and maximum value.

serum autoantibody levels between patients and controls were compared using the Mann-Whitney $U$ test; the positive rates of STIP1 autoantibodies between the patient group and control group or in each group of patients' sera were compared using chi-squared tests. We plotted receiver operating characteristic (ROC) curve to discuss the sensitivity, specificity, and area under the ROC curve (AUC). The cutoff value for autoantibody positivity was determined by achieving the maximum sensitivity when the specificity was more than $90 \%$ and by minimizing the distance of the cutoff value to the top-left corner of the ROC curve (all ESCC versus all normal controls in the training cohort). The test result indicated a statistically significant difference when $P$ value was less than 0.05 (two-sided).

\section{Results}

3.1. Level of Serum Autoantibodies against STIP1. In the group of all ESCC patients in the training cohort, the mean $\mathrm{OD} \pm \mathrm{SD}$ of STIP1 autoantibodies was $0.182 \pm 0.150$ and was $0.161 \pm 0.105$ and $0.102 \pm 0.056$ in the early-stage patient group and normal control group, respectively. We observed that patients with ESCC had a significant increase in level of serum STIP1 autoantibody, compared with normal controls (Figure $1, P<0.0001$ ). As shown in Figure 1, similar result was noted in early-stage ESCC patients $(P<0.0001)$. In the validation cohort, serum STIP1 autoantibody levels were also raised in ESCC patients, compared with controls (Figure 1).

3.2. Diagnostic Performance of Serum Autoantibodies against STIP1. Using ROC analysis (all ESCC group versus control group in the training cohort), we identified a cutoff value of 0.173 for serum autoantibodies against STIP1 to diagnose ESCC (Figure 2). In both cohorts, the positive rates of serum autoantibodies against STIP1 increased not only in patients with ESCC but also in the early-stage patients, compared with normal controls $(P<0.0001$, Table 2$)$. For all ESCC patients in the training cohort, autoantibodies against STIP1 had an AUC of 0.682 (95\% CI: 0.619-0.746) to distinguish individuals with ESCC from normal controls with a sensitivity/specificity of $41.9 \%$ (95\% CI: $33.9 \%-50.3 \%) / 90.1 \%$ (95\% CI: 82.6\%-94.7\%) (Figure 2, Table 3). Autoantibodies against STIP1 also identified early-stage ESCC with a similar AUC value of 0.684 (95\% CI: 0.586-0.782), a 


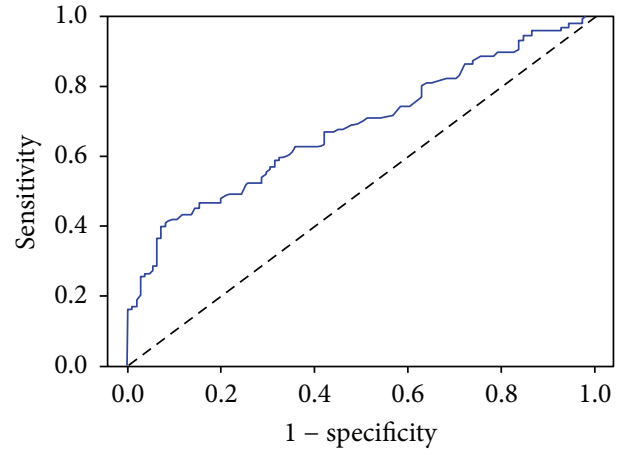

(a)

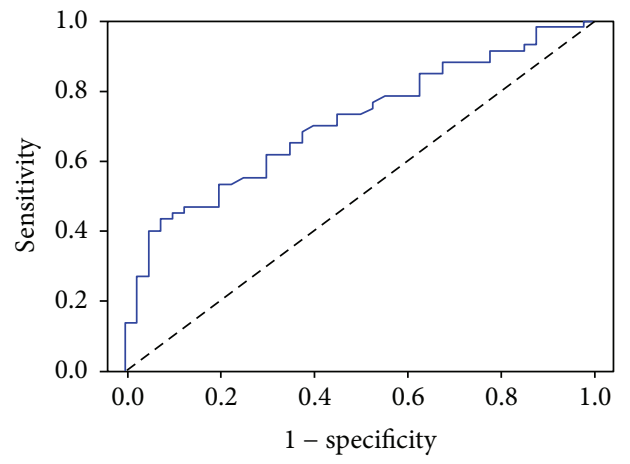

(c)

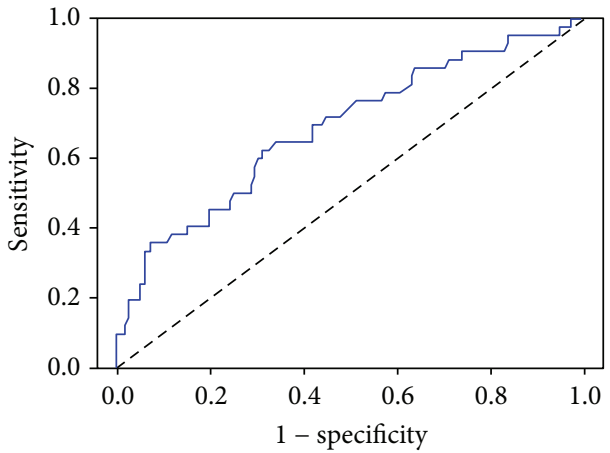

(b)

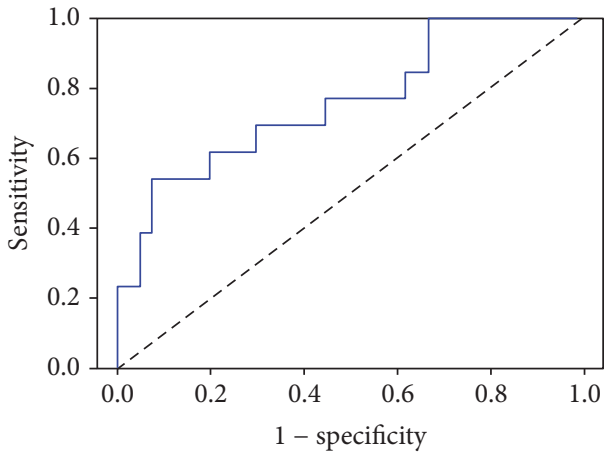

(d)

FIGURE 2: Receiver operating characteristic (ROC) curve analysis in the diagnosis of esophageal squamous cell carcinoma (ESCC). (a) ROC curve for serum autoantibodies against STIP1 for patients with ESCC versus normal controls in the training cohort. (b) ROC curve for serum autoantibodies against STIP1 for patients with early-stage ESCC versus normal controls in the training cohort. (c) ROC curve for serum autoantibodies against STIP1 for patients with ESCC versus normal controls in the validation cohort. (d) ROC curve for serum autoantibodies against STIP1 for patients with early-stage ESCC versus normal controls in the validation cohort.

TABLE 2: Frequency of autoantibodies against STIP1.

\begin{tabular}{lccc}
\hline Group & $N$ & Positive $(\%, 95 \%$ CI $)$ & $P$ value \\
\hline $\begin{array}{l}\text { Training cohort } \\
\quad \text { All ESCC }\end{array}$ & 148 & $62(41.9 \%, 33.9 \%-50.3 \%)$ & $<0.0001$ \\
$\quad \begin{array}{l}\text { Early-stage } \\
\text { ESCC (0+I+ IIA) }\end{array}$ & 42 & $15(35.7 \%, 22.0 \%-52.0 \%)$ & $<0.0001$ \\
$\quad$ Normal controls & 111 & $11(9.9 \%, 5.3 \%-17.4 \%)$ & \\
$\begin{array}{l}\text { Validation cohort } \\
\text { All ESCC }\end{array}$ & 60 & $24(40.0 \%, 27.8 \%-53.5 \%)$ & $<0.0001$ \\
$\quad \begin{array}{l}\text { Early-stage } \\
\text { ESCC (0+I+ IIA) }\end{array}$ & 13 & $5(38.5 \%, 15.1 \%-67.7 \%)$ & $<0.05$ \\
$\quad$ Normal controls & 40 & $3(7.5 \%, 2.0 \%-21.5 \%)$ & \\
\hline
\end{tabular}

ESCC: esophageal squamous cell carcinoma; $P$ value is relative to normal controls. Statistical significance was determined using unpaired chi-square test.

sensitivity of $35.7 \%$ (95\% CI: $22.0-52.0 \%$ ), and a specificity of $90.1 \%$ (95\% CI: $82.6 \%-94.7 \%$ ). In the validation cohort, we found similar diagnostic performance to those from the training cohort when the same cutoff value was used (Table 3). To improve clinical interpretation, we also presented false positive rate, false negative rate, predictive values, and likelihood ratios for autoantibodies against STIP1 in ESCC diagnosis in Table 3.
3.3. Correlation between Autoantibodies against STIP1 and ESCC Clinicopathological Variables. Tables 4 and 5 demonstrate the relationship of the levels of autoantibodies against STIP1 with clinicopathological features in ESCC in the training cohort and validation cohort, respectively. However, serum levels of autoantibodies against STIP1 showed no significant associations with clinicopathologic variables examined, including patient age, patient gender, size of tumor, site of tumor, histological grade, lymph node status, or early-stage and advanced-stage groups (all $P>0.05$ ).

\section{Discussion}

Until now, it has been destitute of efficient early detection approach for ESCC, leading to a postponement of diagnosis and treatment in the majority of patients. To improve longterm survival and life quality of cancer patients, early detection remains the most promising approach. Endoscopic screening has been proven to be a valid method to detect early ESCC and can decrease incidence and mortality of ESCC [26]. But it still could not be widely used as a screening tool for esophageal cancer due to its invasive nature. Therefore, we need to identify serum/plasma biomarkers that could effectively detect early ESCC. In the present study, the serum levels of autoantibodies against STIP1 were significantly increased in ESCC patients compared with normal controls. 


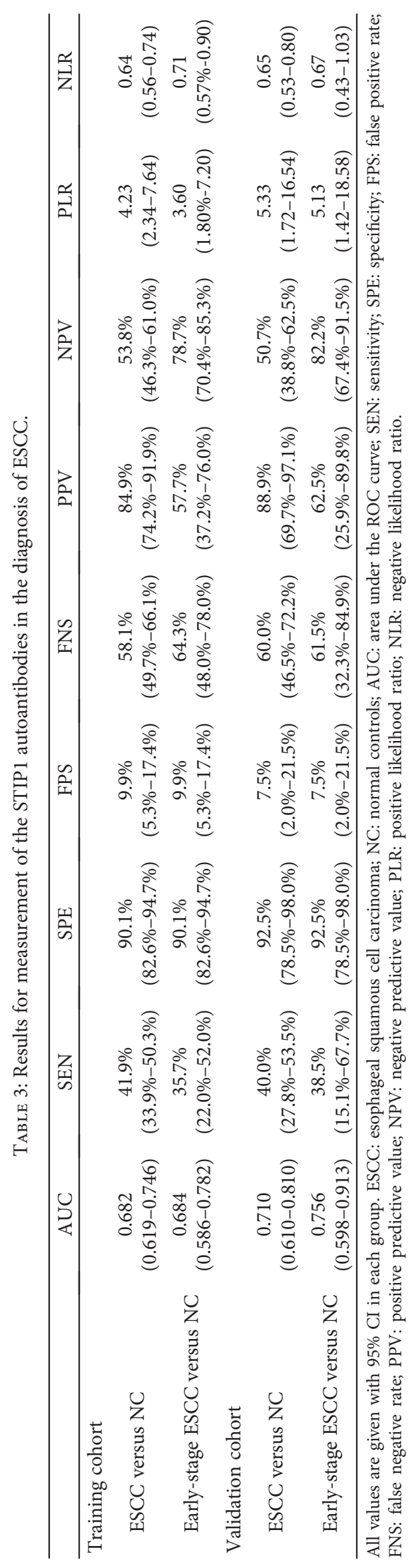


TABLE 4: Relationship between positive rate of the STIP1 autoantibodies and clinical data in ESCC patients from the training cohort.

\begin{tabular}{|c|c|c|c|}
\hline & $N$ & Positive $(\%, 95 \% \mathrm{CI})$ & $P$ \\
\hline \multicolumn{4}{|l|}{ Age } \\
\hline$\geq 58$ & 84 & $40(47.6,36.7-58.7)$ & \multirow[t]{2}{*}{0.106} \\
\hline$<58$ & 64 & $22(34.4,23.3-47.4)$ & \\
\hline \multicolumn{4}{|l|}{ Gender } \\
\hline Male & 114 & $49(43.0,33.8-52.6)$ & \multirow[t]{2}{*}{0.622} \\
\hline Female & 34 & $13(38.2,22.7-56.4)$ & \\
\hline \multicolumn{4}{|l|}{ Smoke } \\
\hline Yes & 106 & $45(42.5,33.0-52.4)$ & \multirow[t]{2}{*}{0.826} \\
\hline No & 42 & $17(40.5,26.0-56.7)$ & \\
\hline \multicolumn{4}{|l|}{ Site of tumor } \\
\hline Upper thorax & 15 & $7(46.7,22.3-72.6)$ & \multirow[t]{3}{*}{0.880} \\
\hline Middle thorax & 101 & $41(40.6,31.1-50.8)$ & \\
\hline Low thorax & 32 & $14(43.8,26.8-62.1)$ & \\
\hline \multicolumn{4}{|l|}{ Size of tumor } \\
\hline$<5 \mathrm{~cm}$ & 71 & $32(45.1,33.4-57.3)$ & \multirow[t]{2}{*}{0.452} \\
\hline$\geq 5 \mathrm{~cm}$ & 77 & $30(39.0,28.3-50.8)$ & \\
\hline \multicolumn{4}{|l|}{ Depth of tumor invasion } \\
\hline $\mathrm{T} 1+\mathrm{T} 2$ & 30 & $9(30.0,15.4-49.6)$ & \multirow[t]{2}{*}{0.133} \\
\hline $\mathrm{T} 3+\mathrm{T} 4$ & 115 & $52(45.2,36.0-54.8)$ & \\
\hline \multicolumn{4}{|l|}{ Regional lymph nodes } \\
\hline No & 77 & $30(39.0,28.3-50.8)$ & \multirow[t]{2}{*}{0.452} \\
\hline $\mathrm{N} 1+\mathrm{N} 2+\mathrm{N} 3$ & 71 & $32(45.1,33.4-57.3)$ & \\
\hline \multicolumn{4}{|l|}{ Histological grade } \\
\hline $\mathrm{G} 1$ & 50 & $18(36.0,23.3-50.9)$ & \multirow[t]{3}{*}{0.440} \\
\hline $\mathrm{G} 2$ & 84 & $39(46.4,35.6-57.6)$ & \\
\hline G3 & 14 & $5(35.7,14.0-64.4)$ & \\
\hline \multicolumn{4}{|l|}{ TNM stage } \\
\hline Early stage $(0+\mathrm{I}+\mathrm{IIA})$ & 42 & $15(35.7,22.0-52.0)$ & \multirow[t]{2}{*}{0.338} \\
\hline $\begin{array}{l}\text { Advanced stage } \\
(\mathrm{IIB}+\mathrm{III}+\mathrm{IV})\end{array}$ & 106 & $47(44.3,34.8-54.3)$ & \\
\hline
\end{tabular}

ESCC: esophageal squamous cell carcinoma; CI: exact confidence interval. Statistical significance was determined using the chi-square test.

In addition, autoantibodies against STIP1 could detect early-stage ESCC (AJCC $0+$ I+ IIA). Our findings indicated autoantibodies against STIP1 as a potential noninvasive biomarker for early ESCC detection.

In recent years, there is no doubt that autoantibodies against TAAs have been a hot topic of research in early cancer diagnosis. Since autoantibodies against TAA in the sera of melanoma patients were first reported [27], a huge number of autoantibodies have been reported as early diagnostic biomarkers for cancers [9-11, 21-23, 28-30]. EarlyCDT-Lung, as a convenient blood test measuring autoantibodies against seven TAAs, shows the ability to aid in risk assessment and the early detection of lung cancer in high-risk, asymptomatic patients $[31,32]$. In ESCC, there are also findings related to measurement of a panel of autoantibodies [22,33]. Our prior work using a panel of six TAAs (i.e., p53, NY-ESO-1, MMP7, Hsp70, PRDX, and Bmi-1) to assess the early-stage ESCC
TABLE 5: Relationship between positive rate of the STIP1 autoantibodies and clinical data in ESCC patients from the validation cohort.

\begin{tabular}{|c|c|c|c|}
\hline & $N$ & Positive (\%, 95\% CI) & $P$ \\
\hline \multicolumn{4}{|l|}{ Age } \\
\hline$\geq 58$ & 32 & $13(40.6,24.2-59.2)$ & \multirow[t]{2}{*}{0.916} \\
\hline$<58$ & 28 & $11(39.3,22.2-59.3)$ & \\
\hline \multicolumn{4}{|l|}{ Gender } \\
\hline Male & 38 & $14(36.8,22.3-54.0)$ & \multirow[t]{2}{*}{0.512} \\
\hline Female & 22 & $10(45.5,25.1-67.3)$ & \\
\hline \multicolumn{4}{|l|}{ Smoke } \\
\hline Yes & 36 & $13(36.1,21.3-53.8)$ & \multirow[t]{2}{*}{0.451} \\
\hline No & 24 & $11(45.8,26.2-66.8)$ & \\
\hline \multicolumn{4}{|l|}{ Site of tumor } \\
\hline Upper thorax & 10 & $3(30.0,8.1-64.6)$ & \multirow[t]{2}{*}{0.724} \\
\hline Middle thorax + low thorax & 50 & $21(42.0,28.5-56.7)$ & \\
\hline \multicolumn{4}{|l|}{ Size of tumor } \\
\hline$<5 \mathrm{~cm}$ & 24 & $9(37.5,19.6-59.2)$ & \multirow[t]{2}{*}{0.747} \\
\hline$\geq 5 \mathrm{~cm}$ & 36 & $15(41.7,26.0-59.1)$ & \\
\hline \multicolumn{4}{|l|}{ Depth of tumor invasion } \\
\hline $\mathrm{T} 1+\mathrm{T} 2$ & 21 & $9(42.9,22.6-65.6)$ & \multirow[t]{2}{*}{0.800} \\
\hline $\mathrm{T} 3+\mathrm{T} 4$ & 38 & $15(39.5,24.5-56.5)$ & \\
\hline \multicolumn{4}{|l|}{ Regional lymph nodes } \\
\hline N0 & 31 & $12(38.7,22.4-57.7)$ & \multirow[t]{2}{*}{0.833} \\
\hline $\mathrm{N} 1+\mathrm{N} 2+\mathrm{N} 3$ & 29 & $12(41.4,24.1-60.9)$ & \\
\hline \multicolumn{4}{|l|}{ Histological grade } \\
\hline G1 & 17 & $6(35.3,15.3-61.4)$ & \multirow[t]{3}{*}{0.659} \\
\hline G2 & 36 & $16(44.4,28.3-61.7)$ & \\
\hline G3 & 7 & $2(28.6,5.1-69.7)$ & \\
\hline \multicolumn{4}{|l|}{ TNM stage } \\
\hline Early stage $(0+\mathrm{I}+\mathrm{IIA})$ & 13 & $5(38.5,15.1-67.7)$ & \multirow[t]{2}{*}{0.898} \\
\hline $\begin{array}{l}\text { Advanced stage } \\
(\mathrm{IIB}+\mathrm{III}+\mathrm{IV})\end{array}$ & 47 & $19(40.4,26.7-55.7)$ & \\
\hline
\end{tabular}

ESCC: esophageal squamous cell carcinoma; CI: exact confidence interval. Statistical significance was determined using the chi-square test.

detection obtained a sensitivity and specificity of $45 \%$ and $95 \%$, respectively [22]. Zhang et al. also reported that autoantibodies against a panel of four TAAs (i.e., c-Myc, HCCR, p53, and p62) provided a high diagnostic efficiency for early-stage ESCC detection [33]. All the abovementioned studies highlight the significance of the combined measurement of an optimized autoantibody panel in the diagnosis of early cancer. However, the two panels of TAAs that have been reported do not have high enough sensitivity as a reliable screening test for early ESCC [22,33]. This study shows that autoantibodies against STIP1 have sensitivities of 35.7\% (95\% CI: $22.0-52.0 \%$ ) in the training cohort and $38.5 \%$ (95\% CI: 15.1\%-67.7\%) in the validation cohort to diagnose early-stage ESCC. Such sensitivity is better than the recently identified autoantibody biomarkers in early-stage ESCC reported by our team, including autoantibodies against ezrin, fascin, and L1CAM $[23,34,35]$, which indicates that autoantibodies against STIP1 might be an encouraging candidate 
for establishment of an optimized autoantibody signature required to gain high sensitivity necessary for ESCC screening. In the next stage of the research, we will further evaluate whether the combination of autoantibodies against STIP1 with other autoantibody targets would increase the diagnostic sensitivity of our previously reported autoantibody panel [22].

To date, there is not yet relevant research of STIP1 in esophageal cancer. This study provides evidence that STIP1 induced autoantibody responses in sera of ESCC patients. It is generally believed that autoantibody production is involved with alterations in expression level, degradation, or posttranslational modification of cellular proteins [36]. Though what mechanism might underlie the production of autoantibodies against STIP1 in esophageal cancer is unclear, our findings provide important clues for further study of biological function of STIP1 in ESCC. In future work, we would perform a systematic study, including in vitro cellbased research and animal experiments, to reveal the function and molecular mechanisms of STIP1 in esophageal cancer. We could suppose that STIP1 might play a role in the carcinogenesis and development of ESCC.

\section{Conclusion}

To our knowledge, this is the first study that shows the elevated levels of serum autoantibodies against STIP1 in ESCC patients. The diagnostic value of serum autoantibodies against STIP1 for ESCC was verified in the training cohort and in an independent validation cohort. Our data demonstrated that autoantibodies against STIP1 might be a potential biomarker significantly associated with ESCC. However, the sample size of patients with early-stage ESCC was relatively small in this study. Further validation of the diagnostic value of autoantibodies against STIP1 in larger sample set is warranted.

\section{Conflicts of Interest}

The authors declare that they have no conflicts of interest.

\section{Authors' Contributions}

Yi-Wei Xu and Can-Tong Liu contributed equally to this work.

\section{Acknowledgments}

This work was supported by the National Science Foundation of China (no. 31600632, no. 81172264, and no. 81472613), the Shantou University Medical College Clinical Research Enhancement Initiative (201428), the Innovative and Strong School Project of Guangdong (2015KQNCX044), and the Department of Education, Guangdong Government under the Top-tier University Development Scheme for Research and Control of Infectious Diseases.

\section{References}

[1] Global Burden of Disease Cancer Collaboration, C. Fitzmaurice, D. Dicker et al., “The global burden of cancer 2013," JAMA Oncology, vol. 1, no. 4, pp. 505-527, 2015.

[2] W. Chen, R. Zheng, P. D. Baade et al., "Cancer statistics in China, 2015," CA: A Cancer Journal for Clinicians, vol. 66, no. 2, pp. 115-132, 2016.

[3] L. A. Torre, F. Bray, R. L. Siegel, J. Ferlay, J. Lortet-Tieulent, and A. Jemal, "Global cancer statistics, 2012," CA: A Cancer Journal for Clinicians, vol. 65, no. 2, pp. 87-108, 2015.

[4] E. Hiripi, L. Jansen, A. Gondos et al., "Survival of stomach and esophagus cancer patients in Germany in the early 21st century," Acta Oncologica, vol. 51, no. 7, pp. 906-914, 2012.

[5] G. Q. Wang, G. G. Jiao, F. B. Chang et al., "Long-term results of operation for 420 patients with early squamous cell esophageal carcinoma discovered by screening," The Annals of Thoracic Surgery, vol. 77, no. 5, pp. 1740-1744, 2004.

[6] K. Mealy, J. Feely, I. Reid, J. McSweeney, T. Walsh, and T. P. Hennessy, "Tumour marker detection in oesophageal carcinoma," European Journal of Surgical Oncology, vol. 22, no. 5, pp. 505-507, 1996.

[7] H. Shimada, Y. Nabeya, S. Okazumi et al., "Prognostic significance of CYFRA 21-1 in patients with esophageal squamous cell carcinoma," Journal of the American College of Surgeons, vol. 196, no. 4, pp. 573-578, 2003.

[8] S. Kosugi, T. Nishimaki, T. Kanda, S. Nakagawa, M. Ohashi, and K. Hatakeyama, "Clinical significance of serum carcinoembryonic antigen, carbohydrate antigen 19-9, and squamous cell carcinoma antigen levels in esophageal cancer patients," World Journal of Surgery, vol. 28, no. 7, pp. 680685, 2004.

[9] E. M. Tan and J. Zhang, "Autoantibodies to tumor-associated antigens: reporters from the immune system," Immunological Reviews, vol. 222, pp. 328-340, 2008.

[10] J. X. Shi, J. J. Qin, H. Ye, P. Wang, K. J. Wang, and J. Y. Zhang, "Tumor associated antigens or anti-TAA autoantibodies as biomarkers in the diagnosis of ovarian cancer: a systematic review with meta-analysis," Expert Review of Molecular Diagnostics, vol. 15, no. 6, pp. 829-852, 2015.

[11] W. Liu, B. Peng, Y. Lu, W. Xu, W. Qian, and J. Y. Zhang, "Autoantibodies to tumor-associated antigens as biomarkers in cancer immunodiagnosis," Autoimmunity Reviews, vol. 10, no. 6, pp. 331-335, 2011.

[12] C. M. Nicolet and E. A. Craig, "Isolation and characterization of STI1, a stress-inducible gene from Saccharomyces cerevisiae," Molecular and Cellular Biology, vol. 9, no. 9, pp. 36383646, 1989.

[13] O. O. Odunuga, V. M. Longshaw, and G. L. Blatch, "Hop: more than an Hsp70/Hsp90 adaptor protein," BioEssays, vol. 26, no. 10, pp. 1058-1068, 2004.

[14] A. B. Schmid, S. Lagleder, M. A. Gräwert et al., "The architecture of functional modules in the Hsp90 co-chaperone Sti1/ Hop," The EMBO Journal, vol. 31, no. 6, pp. 1506-1517, 2012.

[15] H. Kubota, S. Yamamoto, E. Itoh et al., "Increased expression of co-chaperone HOP with HSP90 and HSC70 and complex formation in human colonic carcinoma," Cell Stress \& Chaperones, vol. 15, no. 6, pp. 1003-1011, 2010.

[16] N. Walsh, N. O'Donovan, S. Kennedy et al., "Identification of pancreatic cancer invasion-related proteins by proteomic analysis," Proteome Science, vol. 7, p. 3, 2009. 
[17] J. Padden, D. A. Megger, T. Bracht et al., "Identification of novel biomarker candidates for the immunohistochemical diagnosis of cholangiocellular carcinoma," Molecular \& Cellular Proteomics, vol. 13, no. 10, pp. 2661-2672, 2014.

[18] H. Cho, S. Kim, H. Y. Shin et al., "Expression of stressinduced phosphoprotein1 (STIP1) is associated with tumor progression and poor prognosis in epithelial ovarian cancer," Genes, Chromosomes \& Cancer, vol. 53, no. 4, pp. 277-288, 2014.

[19] A. Chao, C. H. Lai, C. L. Tsai et al., "Tumor stress-induced phosphoprotein1 (STIP1) as a prognostic biomarker in ovarian cancer," PloS One, vol. 8, no. 2, article e57084, 2013.

[20] M. J. Scanlan, J. D. Gordan, B. Williamson et al., "Antigens recognized by autologous antibody in patients with renal-cell carcinoma," International Journal of Cancer, vol. 83, no. 4, pp. 456-464, 1999.

[21] S. Kim, H. Cho, E. J. Nam et al., "Autoantibodies against stress-induced phosphoprotein-1 as a novel biomarker candidate for ovarian cancer," Genes, Chromosomes \& Cancer, vol. 49, no. 7, pp. 585-595, 2010.

[22] Y. W. Xu, Y. H. Peng, B. Chen et al., “Autoantibodies as potential biomarkers for the early detection of esophageal squamous cell carcinoma," The American Journal of Gastroenterology, vol. 109, no. 1, pp. 36-45, 2014.

[23] L. Li, M. Liu, J. B. Lin et al., "Diagnostic value of autoantibodies against ezrin in esophageal squamous cell carcinoma," Disease Markers, vol. 2017, Article ID 2534648, 6 pages, 2017.

[24] T. W. Rice, E. H. Blackstone, and V. W. Rusch, "7th edition of the AJCC Cancer Staging Manual: esophagus and esophagogastric junction," Annals of Surgical Oncology, vol. 17, no. 7, pp. 1721-1724, 2010.

[25] Y. H. Peng, Y. W. Xu, L. S. Huang et al., “Autoantibody signatures combined with Epstein-Barr virus capsid antigen-IgA as a biomarker panel for the detection of nasopharyngeal carcinoma," Cancer Prevention Research, vol. 8, no. 8, pp. 729-736, 2015.

[26] W. Q. Wei, Z. F. Chen, Y. T. He et al., "Long-term follow-up of a community assignment, one-time endoscopic screening study of esophageal cancer in China," Journal of Clinical Oncology, vol. 33, no. 17, pp. 1951-1957, 2015.

[27] H. Shiku, T. Takahashi, L. A. Resnick, H. F. Oettgen, and L. J. Old, "Cell surface antigens of human malignant melanoma. III. Recognition of autoantibodies with unusual characteristics," The Journal of Experimental Medicine, vol. 145, no. 3, pp. 784-789, 1977.

[28] X. Wang, J. Yu, A. Sreekumar et al., "Autoantibody signatures in prostate cancer," The New England Journal of Medicine, vol. 353, no. 12, pp. 1224-1235, 2005.

[29] K. S. Anderson, D. W. Cramer, S. Sibani et al., "Autoantibody signature for the serologic detection of ovarian cancer," Journal of Proteome Research, vol. 14, no. 1, pp. 578-586, 2015.

[30] Y. H. Peng, Y. W. Xu, H. Guo et al., "Combined detection of serum Dickkopf-1 and its autoantibodies to diagnose esophageal squamous cell carcinoma," Cancer Medicine, vol. 5, no. 7, pp. 1388-1396, 2016.

[31] S. Lam, P. Boyle, G. F. Healey et al., "EarlyCDT-Lung: an immunobiomarker test as an aid to early detection of lung cancer," Cancer Prevention Research, vol. 4, no. 7, pp. 1126-1134, 2011.

[32] C. J. Chapman, G. F. Healey, A. Murray et al., "EarlyCDT ${ }^{\circledR}$ Lung test: improved clinical utility through additional autoantibody assays," Tumour Biology, vol. 33, no. 5, pp. 1319-1326, 2012.

[33] H. F. Zhang, J. J. Qin, P. F. Ren et al., "A panel of autoantibodies against multiple tumor-associated antigens in the immunodiagnosis of esophageal squamous cell cancer," Cancer Immunology, Immunotherapy, vol. 65, no. 10, pp. 12331242, 2016.

[34] W. X. Chen, X. B. Hong, C. Q. Hong et al., "Tumor-associated autoantibodies against ascin as a novel diagnostic biomarker for esophageal squamous cell carcinoma," Clinics and Research in Hepatology and Gastroenterology, vol. 41, no. 3, pp. 327332, 2017.

[35] Y. W. Xu, Y. H. Peng, L. Q. Ran et al., "Circulating levels of autoantibodies against L1-cell adhesion molecule as a potential diagnostic biomarker in esophageal squamous cell carcinoma," Clinical \& Translational Oncology, vol. 19, no. 7, pp. 898-906, 2017.

[36] H. T. Tan, J. Low, S. G. Lim, and M. C. Chung, "Serum autoantibodies as biomarkers for early cancer detection," The FEBS Journal, vol. 276, no. 23, pp. 6880-6904, 2009. 


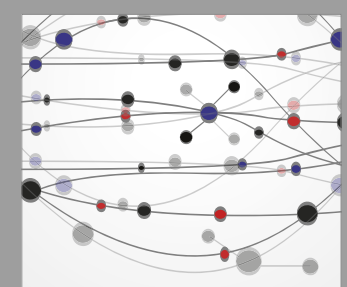

The Scientific World Journal
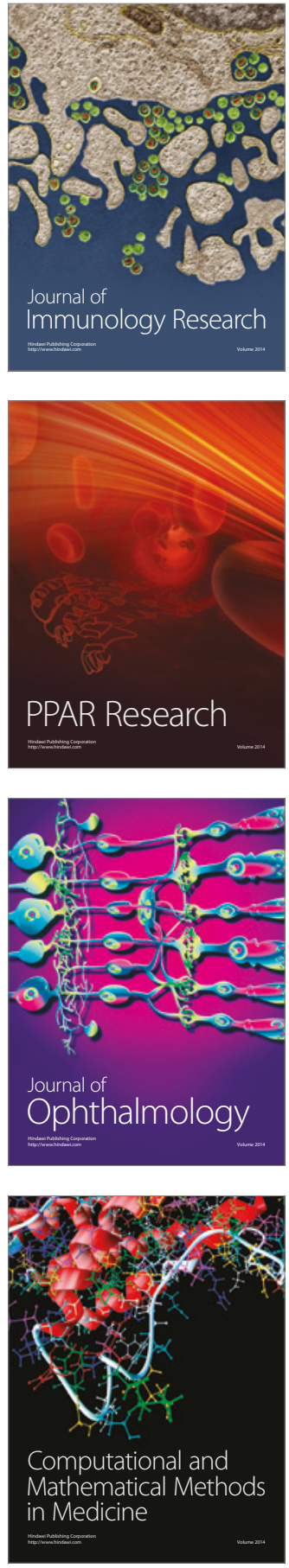

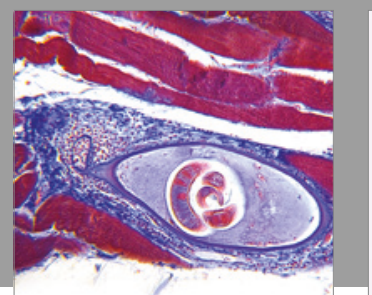

Gastroenterology Research and Practice
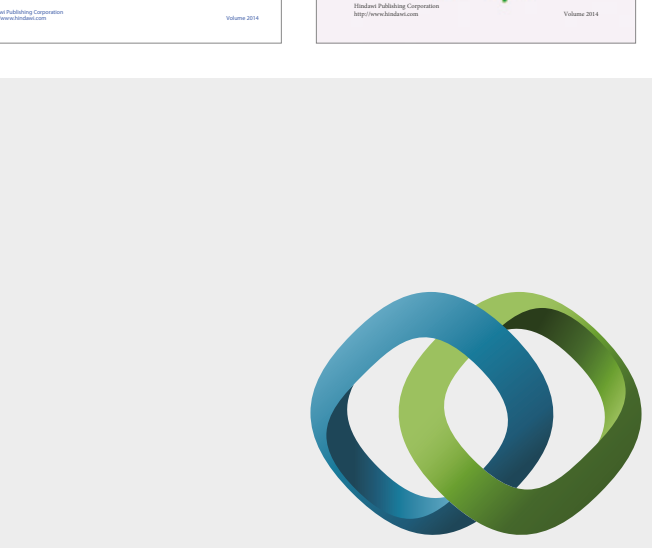

\section{Hindawi}

Submit your manuscripts at

https://www.hindawi.com
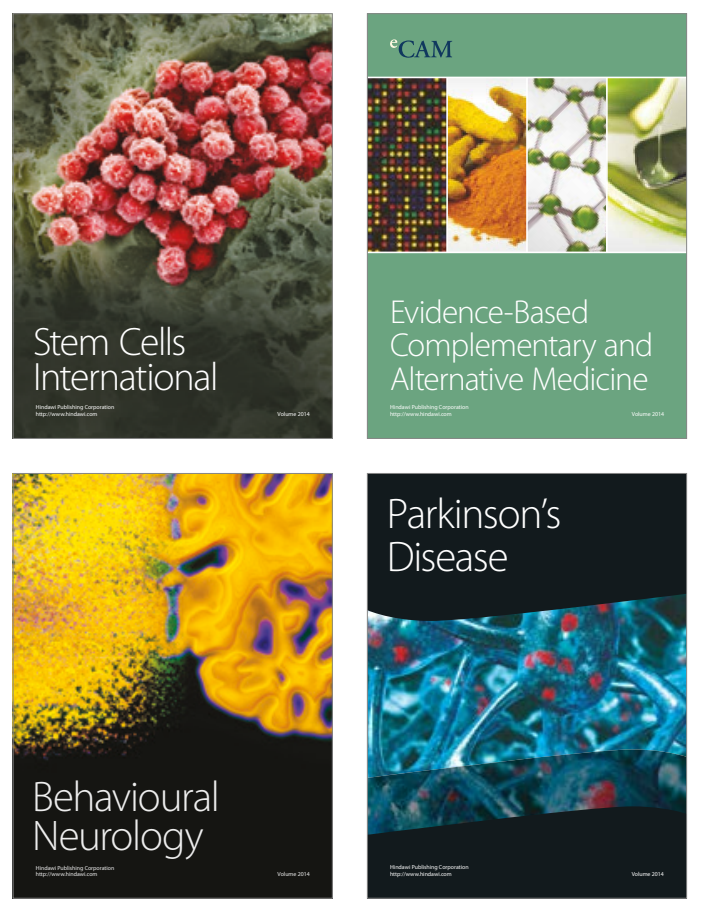
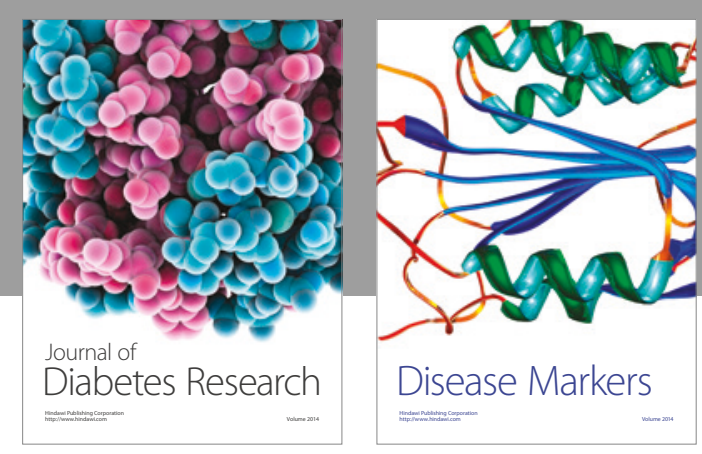

Disease Markers
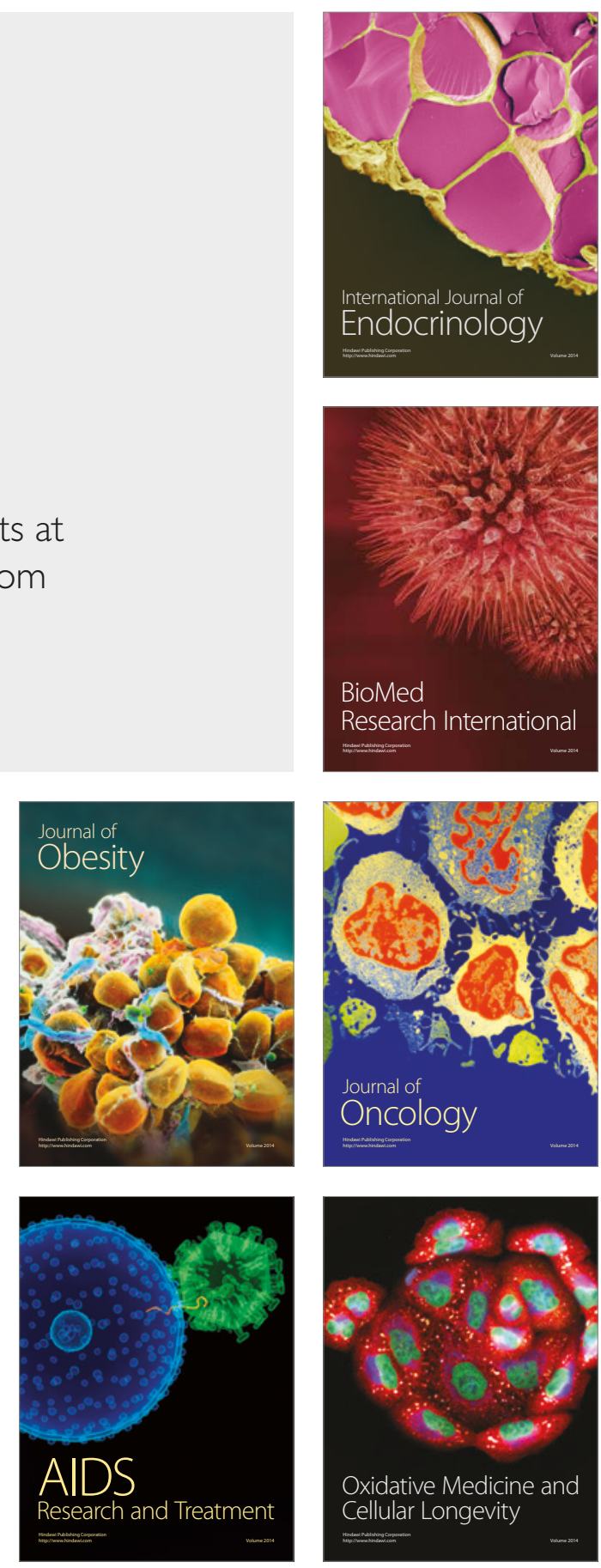